\title{
Noether theorem of a kind of singular integral equation with Hilbert kernel on closed contours
}

\author{
Li-xia Cao \\ Mathematics College \\ Northeast Petroleum University \\ Daqing, Heilongjiang, China \\ caolixia98237@163.com
}

\begin{abstract}
We considered a kind of singular integral equation with Hilbert kernel on closed contours. By using the method of complex functions, we obtain the extended Plemelj Formula with Hilbert kernel, and based on this, we obtained the related conditions of solvability and the general solution for the characteristic singular integral equation with Hilbert kernel on closed contours.
\end{abstract}

Keywords-Hilbert kernel; singular integral equation; general solution; solvable conditions

\section{INTRODUCTION}

In $[1,2,3,4,5]$, the authors discussed the singular integral equation with Cauchy kernel on the real half-line, on the real line, or on a complicated contour. In [6], the authors discussed Numerical solution of a singular integral equation with Cauchy kernel in the plane contact problem. While the solvable Noether theorem for the singular integral equations with Hilbert kernel, has few been discussed. Here we study a kind of singular integral equation with Hilbert kernel on closed contours by transforming it into the periodic Riemann boundary value problem, then give the general solutions and the solvable conditions.

\section{THE CHARACTERISTIC SINGULAR INTEGRAL EQUATION WITH HILBERT KERNEL}

Suppose that $L=\sum_{j=1}^{p} L_{j}$ is a finite set of nonintersecting simple closed contours, which is nonintersecting under translation along the real axis by $a \pi(a>0)$. Without loss of generality, we assume that $L_{j}$ is oriented in the counterclockwise direction and not passing through $Z= \pm a \pi / 2$ and their periodic points. We assume that $A(t), B(t), f(t) \in H(L)$ are the given functions, and that $A^{2}(t)-B^{2}(t) \neq 0$ on $L$. We consider the following the characteristic singular integral equation with Hilbert kernel for a Hölder continuous function $\varphi(t)$ on $L$

$$
K^{0} \varphi \equiv A\left(t_{0}\right) \varphi\left(t_{0}\right)+\frac{B\left(t_{0}\right)}{a \pi i} \int_{L} \cot \frac{t-t_{0}}{a} \varphi(t) d t, t_{0} \in L \text {. }
$$

\section{TRANSFORMATION OF GIVEN EQUATION}

Lemma 1 (Plemelj Formula with Hilbert kernel) suppose that $L$ is a closed or open smooth curve. If $g(t) \in H$ with period $a \pi(a>0)$, and

$$
\Psi(z)=\frac{1}{2 a \pi i} \int_{L} g(t) \cot \frac{t-z}{a} \mathrm{~d} t, z \notin L, z \notin L .
$$

Then

$$
\Psi^{ \pm}\left(t_{0}\right)= \pm \frac{1}{2} g\left(t_{0}\right)+\frac{1}{2 a \pi i} \int_{L} g(t) \cot \frac{t-t_{0}}{a} \mathrm{~d} t, t_{0} \in L
$$

Proof. With the aid of the Laurent series expansion of $\cot [(\tau-t) / a]$, we can obtain the Lemma by using similar method in [1].

Now we aim to transform the given equation into Riemann boundary value problem. Let

$$
\Phi(z)=\frac{1}{2 a \pi i} \int_{L} \varphi(t) \cot \frac{t-z}{a} d t,
$$

then $\Phi(z)$ is a sectionally holomorphic function with period $a \pi$, and with jump $L$. From the extended Plemelj formula with Hilbert kernel (2), we have

$$
\varphi\left(t_{0}\right)=\Phi^{+}\left(t_{0}\right)-\Phi^{-}\left(t_{0}\right), t_{0} \in L,
$$

Eq.(2) can be transformed into the PR problem (period Riemann boundary problem)

$$
\Phi^{+}\left(t_{0}\right)=G\left(t_{0}\right) \Phi^{-}\left(t_{0}\right)+g\left(t_{0}\right), t_{0} \in L,
$$

where

$$
G\left(t_{0}\right)=\frac{A\left(t_{0}\right)-B\left(t_{0}\right)}{A\left(t_{0}\right)+B\left(t_{0}\right)}, g\left(t_{0}\right)=\frac{f\left(t_{0}\right)}{A\left(t_{0}\right)+B\left(t_{0}\right)} .
$$

By noting that

$$
\lim _{z \rightarrow \pm \infty i} \cot \frac{t-z}{a}= \pm i
$$

we conclude that

$$
\Phi( \pm \infty i)= \pm \frac{1}{2 a \pi} \int_{L} \varphi(t) \mathrm{d} t \text { (finite), }
$$

so we have

$$
\Phi(-\infty i)=-\Phi(+\infty i)
$$


Conversely, if $\Phi(z)$ is a sectionally holomorphic function with period $a \pi$ with jump $L$ and satisfy the boundary condition (5) and the additive condition (7), then the function $\varphi\left(t_{0}\right)$ determined by (4) is actually the solution of Eq.(1). Therefore, we obtain the following theorem.

Theorem 3.1 Eq.(1) is equivalent to the PR problem (5) with an additive condition (7), moreover the solution of Eq.(1) is given by (4) whenever the PR problem (5) is solved out.

\section{THE SOLVING OF THE CHARACTERISTIC SINGULAR INTEGRAL EQUATION}

Let $S_{j}^{+}$denote the domain closed by $L_{j}$, and $S_{j}^{-}$denote the domain outside $\overline{S_{j}^{+}}$. By noting that

$$
\pm \infty i \in S_{j}^{-}(j=1,2, \cdots, p)
$$

we may let

$$
\kappa_{j}=\frac{1}{2 \pi i}[\arg G(T)]_{L_{j}},
$$

then

$$
\kappa=\sum_{j} \kappa_{j}
$$

is called the index of Eq. (1).

The canonical function of Eq. (1) can be taken as

$$
X(z)=X_{1}(z) X_{2}(z) \cdots X_{p}(z),
$$

where

$$
X_{j}(z)=\left\{\begin{array}{l}
X_{j}^{+}(z)=e^{\Gamma_{j}(z)}, z \in S_{j}^{+} ; \\
X_{j}^{-}(z)=e^{\Gamma_{j}(z)} /\left(\tan \frac{z}{a}-\tan \frac{c_{j}}{a}\right)^{K_{j}}, z \in S_{j}^{-},
\end{array} \quad j=1,2, \cdots, p,\right.
$$

and

$$
\Gamma(z)=\frac{1}{2 a \pi i} \int_{L_{j}} G(t) \cot \left(\frac{t-z}{a}\right) /\left(\tan \frac{z}{a}-\tan \frac{C_{j}}{a}\right)^{K_{j}} \mathrm{~d} t,
$$

Then by calculation, we have

$$
G_{\infty}=X(-\infty i) / X(+\infty i)=(-1)^{\kappa} e^{-i v},
$$

where

$$
v=\sum_{j=1}^{p} \frac{1}{a \pi i} \int_{L_{j}} \log \left[G(t) /\left(\tan \frac{t}{a}-\tan \frac{Z_{j}}{a}\right)^{\kappa_{j}}\right] \mathrm{d} t-\frac{2}{a} \sum_{j=1}^{p} \kappa_{j} Z_{j} .
$$

Here $\kappa$ may be even or odd, we may talk only for the case that $\kappa$ is even since when $\kappa$ is odd we may change $v$ into $v+\pi$ in the following.

Now we assume that $K$ is even. Let

$Z(t)=[A(t)+B(t)] X^{+}(t)=[A(t)-B(t)] X^{-}(t)$, then we obtain by calculation and arrangement that

$1^{\circ}$ when $\kappa>0$, the general solution of Eq.(1) is

$$
\varphi\left(t_{0}\right)=K^{*} f+B^{*}\left(t_{0}\right) Z\left(t_{0}\right) P_{\kappa}\left(\tan \frac{t_{0}}{a}\right),
$$

where

$$
\begin{aligned}
K^{*} f=A^{*}\left(t_{0}\right) f\left(t_{0}\right)-\frac{B^{*}\left(t_{0}\right) Z\left(t_{0}\right)}{a \pi i} \int_{L} \frac{f(t)}{Z(t)} \cot \frac{t-t_{0}}{a} \mathrm{~d} t, \\
f_{0}=\frac{1}{a \pi i} \int_{L} \frac{f(t)}{Z(t)} \mathrm{d} t, \\
A^{*}(t)=A(t) /\left[A^{2}(t)-B^{2}(t)\right], \\
B^{*}(t)=B(t) /\left[A^{2}(t)-B^{2}(t)\right], \\
P_{\kappa}(w)=c_{0}+c_{1} w+c_{2} w^{2}+\cdots+c_{\kappa} w^{\kappa},
\end{aligned}
$$

and derived from (7), the coefficients of the polynomial $P_{\kappa}(w)$ satisfy the additive condition

$$
-\left(c_{0}-c_{2}+\cdots\right) \cos \frac{v}{2}+\left(c_{1}-c_{3}+\cdots\right) \sin \frac{v}{2}=f_{0} \sin \frac{v}{2} \text {. }
$$

Thus there are $\kappa$ independent constants in the solution given by (11).

$2^{\circ}$ when $\kappa=0$, the additive condition (7) becomes

$$
c_{0} \cos \frac{v}{2}=-f_{0} \sin \frac{v}{2},
$$

and now there are also two cases:

if $v \neq(2 k+1) \pi$, Eq.(1) has unique solution

$$
\varphi\left(t_{0}\right)=K^{*} f-f_{0} \tan \frac{v}{2} B^{*}\left(t_{0}\right) Z\left(t_{0}\right),
$$

and if $v=(2 k+1) \pi$, Eq.(1) has unique solution

$$
\varphi\left(t_{0}\right)=K^{*} f+C \cdot B^{*}\left(t_{0}\right) Z\left(t_{0}\right),
$$

where $C$ is an arbitrary real constant.

$3^{\circ}$ when $\kappa<0$, if and only if the solvable conditions (when $\kappa=-1$, the solvable condition is not required )

$$
\int_{L} \frac{f(t)}{Z(t)}\left(\tan ^{j-1} \frac{t}{a}+\tan ^{j+1} \frac{t}{a}\right) d t=0, j=1, \cdots,-\kappa-1,
$$

and the additive condition

$$
\int_{L} \frac{f(t)}{Z(t)} \sin \left(\frac{t}{a}-\frac{v}{2}\right) /\left(\cos \frac{t}{a}\right) d t=0,
$$

are satisfied, Eq.(1) has unique solution

$$
\varphi\left(t_{0}\right)=K^{*} f-f_{1} B^{*}\left(t_{0}\right) Z\left(t_{0}\right),
$$

where 


$$
f_{r}=\int_{L} \frac{f(t)}{Z(t)} \tan ^{r} \frac{t}{a} \mathrm{~d} t
$$

Combining (19) and (20), solvable conditions can be rewritten as

$$
\left\{\begin{array}{l}
\int_{L} \frac{f(t)}{Z(t)}\left(\tan ^{j-1} \frac{t}{a}+\tan ^{j+1} \frac{t}{a}\right) d t=0, j=1, \cdots,-\kappa-1, \\
\int_{L} \frac{f(t)}{Z(t)}\left(\sin \frac{v}{2}-\cos \frac{v}{2} \tan \frac{t}{a}\right) d t=0 .
\end{array}\right.
$$

\section{THE SOLVING OF THE ASSOCIATIVE EQUATION}

The associative homogeneous of Eq.(1) is

$$
K^{\circ} \psi \equiv A\left(t_{0}\right) \psi\left(t_{0}\right)-\frac{\int_{L} B(t) \psi(t) \cot \frac{t-t_{0}}{a} \mathrm{~d} t}{a \pi i}=0, t_{0} \in L .
$$

It is clearly that the index of Eq.(24) is $\kappa^{\prime}=-\kappa$.

Similar to the solving of the characteristic singular integral equation, we can obtain that when $\kappa^{\prime}>0$, Eq.(24) has the general solution

$$
P_{\kappa^{\prime}}\left(\tan \frac{t_{0}}{a}\right) / Z\left(t_{0}\right),
$$

where

$$
P_{\kappa^{\prime}}(w)=b_{0}+b_{1} w+\cdots+b_{\kappa^{\prime}} w^{\kappa^{\prime}}
$$

is an arbitrary polynomial of degree $\boldsymbol{K}^{\prime}$, and its coefficients satisfies the additive condition

$$
-\left(b_{0}-b_{2}+\cdots\right) \cos \frac{v}{2}+\left(b_{1}-b_{3}+\cdots\right) \sin \frac{v}{2}=0 .
$$

Therefore, we can take a set of linearly independent solutions of Eq.(24) as follows

$$
\begin{gathered}
\left(\sin \frac{v}{2} \tan ^{0} \frac{t}{a}-\cos \frac{v}{2} \tan ^{1} \frac{t}{a}\right) / Z(t),\left(\tan ^{0} \frac{t}{a}+\tan ^{2} \frac{t}{a}\right) / Z(t), \\
\left(\tan ^{1} \frac{t}{a}+\tan ^{3} \frac{t}{a}\right) / Z(t),\left(\tan ^{2} \frac{t}{a}+\tan ^{4} \frac{t}{a}\right) / Z(t),
\end{gathered}
$$

$$
\begin{gathered}
\cdots,\left(\tan ^{\kappa^{\prime}-3} \frac{t}{a}+\tan ^{\kappa^{\prime}-1} \frac{t}{a}\right) / Z(t), \\
\left(\tan ^{\kappa^{\prime}-2} \frac{t}{a}+\tan ^{\kappa^{\prime}} \frac{t}{a}\right) / Z(t) .
\end{gathered}
$$

VI. NOETHER THEOREM OF CHARACTERISTIC SINGULAR INTEGRAL EQUATION WITH HILBERT KERNEL

By comparing the results in IV and $\mathrm{V}$, we can establish the following results.

\section{Theorem 6.1 (Noether Theorem)}

(a) The number of the linearly independent solutions of $K^{0} \varphi=0$ and $K^{\circ} \psi=0$ are all finite, denoted by $l$ and $l^{\prime}$ respectively. Moreover we have

$$
l-l^{\prime}=\kappa,
$$

where $\kappa$ is the index of operator $K^{0}$.

(b) $K^{0} \varphi=f$ is solvable if and only if the conditions

$$
\int_{L} \psi_{k}(t) f(t) d t=0, \quad k=0,1, \cdots, l^{\prime}
$$

are satisfied, where $\left\{\psi_{k}\right\}_{1}^{l^{\prime}}$ is the set of linearly independent solutions of $K^{\circ} \psi=0$.

\section{REFERENCES}

[1] Lu J K.: Boundary Value Problems for Analytic Functions. World Sci Publ, Singapore, 2004

[2] D. Pylak; R. Smarzewski; M. A. Sheshko, A Singular Integral Equation with a Cauchy Kernel on the Real Half-Line, J. Differential Equations, vol. 41, pp. 1775-1788, 2005.

[3]B. G. Gabdulkhaevt; I. N. Tikhonov, Methods for Solving a Singular Integral Equation with Cauchy Kernel on the Real Line,J. Differential equations, vol. 44, pp. 980-990, 2008.

[4] M. A. Sheshko; S. M. Sheshko, Singular integral equation with Cauchy kernel on the real axis, J. Differential Equations, vol. 46, pp. 568-585, 2010. [5] M. A. Sheshko; S. M. Sheshko, Singular integral equation with Cauchy kernel on a complicated contour, Differential Equations, vol. 47, pp. 13441356, 2011 .

[6] M. R. Capobianco; G. Criscuolo, Numerical solution of a singular integral equation with Cauchy kernel in the plane contact problem,J. Quarterly of Applied Mathematics, vol. 69, pp. 79-89, 2011. 